\title{
EXAME ELETROCARDIOGRÁFICO EM EQUINOS DA RAÇA PURO SANGUE ÁRABE SUBMETIDOS AO EXERCÍCIO EM ESTEIRA DE ALTA VELOCIDADE E À SUPLEMENTAÇÃO COM VITAMINA E
}

\section{Electrocardiographic exam in Arabian horses submitted to exercise on high-speed treadmill and vitamin $E$ supplementation}

\author{
YONEZAWA, L.A. ${ }^{1}$; MACHADO, L.P. ${ }^{2}$; SILVEIRA, V.F. ${ }^{3}$; WATANABE, M.J. ${ }^{1}$; \\ SAITO, M.E. ${ }^{4}$; KITAMURA, S.S. ${ }^{5}$; KOHAYAGAWA, A. ${ }^{1}$

\footnotetext{
${ }^{1}$ Faculade de Medicina Veterinária e Zootecnia, Unesp, Botucatu, SP.

${ }^{2}$ Universidade Federal do Piauí (UFPI), Bom Jesus, PI.

${ }^{3}$ Universidade Federal do Recôncavo da Bahia, Cruz das Almas, BA.

${ }^{4}$ Universidade do Estado de Santa Catarina (UDESC), Lages, SC.

${ }^{5}$ Universidade Anhembi-Morumbi, São Paulo, SP.
}

Avaliou-se o efeito do exercício físico, treinamento e suplementação com vitamina $\mathrm{E}$ sobre os parâmetros eletrocardiográficos, utilizando oito éguas da raça Puro Sangue Árabe, sem treinamento prévio, distribuídos em dois grupos: controle $(n=4)$ e suplementado com vitamina $E$ $(n=4)$ na dose diária de 1.000 UI. Os animais foram submetidos a uma prova de exercício progressivo ( $\mathrm{P} 1$ ) em esteira de alta velocidade inclinada a $+7 \%$, em seguida a um período de treinamento de 20 dias e posteriormente uma nova prova de exercício progressivo (P2). Realizouse a análise e interpretação dos traçados eletrocardiográficos quanto ao ritmo, frequência cardíaca, duração e amplitude das ondas e intervalos, antes, imediatamente após e 30 min após o exercício progressivo. Observou-se uma diminuição da frequência cardíaca de repouso após o treinamento. Não houve efeito da suplementação com vitamina $E$ sobre os parâmetros eletrocardiográficos. Os resultados indicaram que o eletrocardiograma foi eficiente na detecção das alterações cardíacas promovidas pelo exercício físico, mas necessita-se de mais estudos para elucidar o seu significado clínico.

Palavras-chave: antioxidante; cavalo; eletrocardiograma; exercício físico

\section{ABSTRACT}

The effect of physical exercise, training and vitamin $E$ supplementation on electrocardiographic parameters was evaluated in eight untrained Arabian mares, divided into two groups: control $(n=4)$ and supplemented with vitamin $E(n=4)$ at the daily dose of $1.000 \mathrm{UI}$. Animals were submitted to an incremental exercise test (P1) on high-speed treadmill inclined $+7 \%$, after that to a training period of 20 days and later to a new incremental exercise test (P2). Analysis and interpretation of electrocardiographic tracings were performed regarding the rhythm, heart rate, duration and amplitude of waves and intervals, before, immediately after and $30 \mathrm{~min}$ after the incremental exercise. A reduction of the rest heart rate was observed after training. There was no effect of vitamin $\mathrm{E}$ supplementation on electrocardiographic parameters. Results indicated that the electrocardiogram was efficient in detention of cardiac alterations promoted by the physical exercise, but more studies are needed to elucidate its clinical meaning. 


\section{INTRODUÇÃO}

O exercício extremo em humanos é amplamente conhecido por apresentar uma variedade de riscos à saúde. Embora não se considere a disfunção miocárdica como um risco, observa-se que, com a participação em eventos cada vez mais exaustivos, o conhecimento da resposta cardíaca tornou-se essencial para a segurança do atleta (Douglas et al., 1987). $\mathrm{Na}$ medicina equina, há a preocupação com a queda de desempenho em decorrência às disfunções cardiovasculares, responsáveis por alterações que causam desde uma intolerância ao exercício até a morte súbita (Kiryu et al., 1999). Do mesmo modo, a suplementação de antioxidantes para melhoria do desempenho atlético ainda necessita ser comprovada (De Moffarts et al., 2005).

Em equinos, as anormalidades cardíacas são referidas como a terceira maior causa de queda de desempenho em atletas, após as alterações músculoesqueléticas e respiratórias (Martin et al., 2000). Entretanto, a disfunção miocárdica causada pelo exercício parece ser de difícil avaliação (Durando et al., 2006). Os mecanismos intercorrentes do exercício físico responsáveis pela lesão miocárdica e disfunção cardíaca resultante ainda precisam ser elucidados. Especula-se que esta lesão possa ocorrer em decorrência de uma isquemia durante exercício, que pode danificar as membranas celulares do miocárdio (Whyte et al., 2000; Neumayr et al., 2002). Igualmente, esses mecanismos de injúria miocárdica podem causar uma instabilidade elétrica e aumento do potencial arritmogênico do músculo cardíaco (Leroux et al., 1995). Sabe-se que a capacidade antioxidante no coração parece ser limitada, o que o torna susceptível à lesão tecidual por estresse oxidativo após um período de exercício agudo (Ascensão et al., 2003). Em contrapartida, a suplementação com vitamina $E$ parece influenciar a resposta tecidual do músculo cardíaco em fenômenos de isquemia-reperfusão, assumindo um papel determinante na proteção do coração contra situações deletérias decorrentes do exercício agudo (Venditti et al. 1999).

O eletrocardiograma é um método de diagnóstico para investigação de processos cardíacos, cujo papel fundamental é a avaliação da atividade elétrica do coração que pode interferir no desempenho atlético dos animais (White e Rhode, 1974; Reef, 1999). Além do baixo custo, é um exame não invasivo e de fácil realização (Fregin, 1982; Fernandes et al., 2004). Na medicina equina, é utilizado para verificar a existência de arritmias e avaliar o efeito do treinamento. Segundo Jesty e Reef (2006), as arritmias cardíacas no equino são muito comuns, mas a maioria é benigna. Algumas arritmias aparecem por um breve intervalo imediatamente após o exercício moderado em muitos animais. Embora o sistema nervoso autonômico provavelmente esteja envolvido na gênese de muitas arritmias, as lesões orgânicas são a causa de muitas outras (Senta et al., 1970).

Há evidências de que a "síndrome do coração atleta" descrita em humanos ocorre no equino, referindo-se às adaptações fisiológicas que podem ser confundidas com doenças cardíacas ou limitações do desempenho atlético dos animais (Evans, 1999). Essa síndrome representa uma adaptação reversível, estrutural e funcional do tecido miocárdico, provocado pelo longo e regular treinamento físico. A resposta fisiológica ao exercício físico é caracterizada tanto por modificações transitórias como ajustes estáveis em consequência ao condicionamento do sistema cardiovascular em resposta aos programas de treinamento (Fazio et al., 2003). Skarda et al. (1976) avaliaram a influência do treinamento sobre a frequência cardíaca, duração dos intervalos PQ e QT e do complexo QRS, no repouso e após exercício submáximo 
padronizado, durante nove semanas de treinamento em equinos inicialmente sedentários. Entretanto, não observaram alterações significativas que comprovassem uma melhora do estado atlético com o treinamento. Costa et al. (1985) observaram, em equinos adultos sem treinamento, valores dos parâmetros eletrocardiográficos similares às médias de animais treinados, porém no limite inferior. Chabchoub et al. (1999) observaram diferenças na duração da onda T, de QRS e no intervalo QT entre equinos considerados de alto e baixo desempenho.

O significado clínico das alterações eletrocardiográficas durante e após o exercício ainda é desconhecido para a espécie equina. Por ser muito lábil, a avaliação do eletrocardiograma é extremamente difícil. Por essa razão, é importante definir as características eletrocardiográficas que se modificam com 0 treinamento, permitindo a diferenciação dos animais que podem apresentar miocardite ou outras alterações patologicas (Muñoz et al., 1995). Portanto, o presente estudo objetivou a análise e interpretação do eletrocardiograma de equinos da raça Puro Sangue Árabe, submetidos ao exercício progressivo em esteira de alta velocidade, ao treinamento e à suplementação com vitamina $\mathrm{E}$.

\section{MATERIAL E MÉTODOS}

Foram utilizadas oito éguas adultas, da raça Puro Sangue Árabe, sem treinamento prévio, com idade variando de 4,5 a 12 anos e peso médio de $383 \mathrm{~kg}$, clinicamente sadias e selecionadas mediante exames hematológico, bioquímico sérico, videoendoscopia do aparelho respiratório anterior com 0 animal em repouso, laringoscopia com o animal em movimento, cinemática do aparelho locomotor, exame eletrocardiográfico de repouso e ecocardiografia bidimensional completa, modo-M e Doppler colorido.

Os animais foram mantidos em piquetes, sob as mesmas condições de manejo alimentar, sanitário e de treinamento. O manejo nutricional consistiu de alimentação à base de feno de capim coast-cross, ração e suplemento mineral comercial para equinos, e água ad libitum, conforme recomendações de Lewis (2000). A medicação anti-helmíntica foi administrada por via oral a cada oito semanas (Klei, 1997). Os equinos foram distribuídos em dois grupos de quatro animais: Controle (GC) e Suplementado com vitamina E (dlalfa-tocoferol) (GE). A suplementação no grupo GE teve início 15 dias antes da primeira prova de exercício na dose de $1.000 \mathrm{UI} /$ animal, via oral, diariamente, sem interrupção até o final do experimento.

Foram realizadas duas provas de exercício progressivo, denominadas de $\mathrm{P} 1$ (antes do treinamento) e P2 (após o treinamento), as quais foram realizadas em esteira de alta velocidade inclinada a $+7 \%$. O exercício iniciou-se a $5 \mathrm{~min}$ a $1,8 \mathrm{~m} / \mathrm{s}$ (passo), seguindo de $3 \mathrm{~min}$ a $4,0 \mathrm{~m} / \mathrm{s}$ (trote), $2 \mathrm{~min}$ a $6,0 \mathrm{~m} / \mathrm{s}$ (cânter) e em seguida $1 \mathrm{~min}$ a $8,0 \mathrm{~m} / \mathrm{s}, 9,0 \mathrm{~m} / \mathrm{s}, 10,0 \mathrm{~m} / \mathrm{s}$, $11,0 \mathrm{~m} / \mathrm{s}$ (galope) ou até quando o animal pudesse manter-se em exercício, mesmo sendo estimulado (Machado et al., 2007).

Entre P1 e P2 houve intervalo, no qual os animais foram submetidos a um período de treinamento, realizado uma vez ao dia, seis vezes por semana com um dia sem realizar trabalho, até completarem 20 dias. O protocolo de treinamento adotado foi de $5 \mathrm{~min}$ a $1,8 \mathrm{~m} / \mathrm{s}$ (passo), $3 \mathrm{~min}$ a 4,0 $\mathrm{m} / \mathrm{s}$ (trote), $2 \mathrm{~min}$ a $6,2 \mathrm{~m} / \mathrm{s}$ (cânter), $1 \mathrm{~min}$ a $8,0 \mathrm{~m} / \mathrm{s}$ e a $10,0 \mathrm{~m} / \mathrm{s}$ (galope), seguidos de um período de desaquecimento a 3,0 $\mathrm{m} / \mathrm{s}$ (trote) por $2 \mathrm{~min}$ e a $1,6 \mathrm{~m} / \mathrm{s}$ (passo) por 2 min (Machado et al., 2007). Após o último dia de treinamento, os animais não foram submetidos à atividade física por 48 $\mathrm{h}$ antes de realizarem a $\mathrm{P} 2$.

O exame eletrocardiográfico foi realizado antes (M0), imediatamente após (PE) e 30 min após o exercício nas provas P1 e P2. Procedeu-se em ambiente fechado sem estímulos externos, com o 
animal mantido em tronco de contenção com piso revestido por placas de borracha para evitar interferências no traçado. Os eletrodos foram fixados à pele por meio de clipes tipo "jacaré" com algodão embebido de álcool. Os traçados foram obtidos, segundo a técnica descrita por Fregin (1982) e por meio de eletrocardiógrafo, registrados e padronizados com sensibilidade de $1 \mathrm{mV}=1 \mathrm{~cm}$ na derivação bipolar DII, em velocidade de $25 \mathrm{~mm} / \mathrm{s}$ para avaliação do ritmo e frequência cardíaca, e em $50 \mathrm{~mm} / \mathrm{s}$ para mensurar a duração e amplitude das ondas e intervalos.

De acordo com a distribuição dos dados analisados pelo Teste de Kolgomorov e Smirnov, as variáveis foram avaliadas segundo métodos estatísticos paramétricos ou não-paramétricos. Na ocorrência do primeiro caso, os dados foram inicialmente avaliados através de teste $F$ de análise de variância, e quando significativo, as médias foram confrontadas pelo teste de múltipla amplitude de Tukey (Sampaio, 1998). No caso de testes nãoparamétricos os mesmos foram analisados pelo teste de Kruskal-Wallis (Siegel, 1995). Todas as análises foram consideradas significativas quando $\mathrm{P}<0,05$.

\section{RESULTADOS}

Os dados referentes aos parâmetros eletrocardiográficos estão apresentados em conjunto (oito animais) na Tabela 1, uma vez que não houve efeito da suplementação com vitamina $E(P>0,05)$ entre os grupos controle e suplementado.

A frequência cardíaca aumentou significativamente após o exercício e não retornou aos valores de repouso após 30 min do exercício, em ambas as provas. Houve diminuição significativa com 0 treinamento, mas apenas na frequência de repouso. Observou-se redução significativa, em ambas as provas, dos intervalos PR e QT, imediatamente após o exercício, retornando aos valores próximos aos de repouso em 30 min no pré-treinamento, mas não no pós. Não houve alterações significativas da duração de QRS e das amplitudes de Q e R.

$E m$ relação às ondas $P$ e $T$, não houve alterações na duração após 0 exercício, nem com o treinamento. Entretanto, a morfologia de ambas variou com o exercício. Na prova $\mathrm{P} 1$, três animais apresentaram a morfologia da onda $P$ monofásica positiva $(+)$, e nos cinco restantes, bífida e positiva (+/+). Logo após o término do exercício, observou-se alteração na polaridade da onda $\mathrm{P}$, pela ocorrência da forma difásica, com 0 componente PI negativo e PII positivo $(-/+)$ em sete animais (Figura 1). Após $30 \mathrm{~min}$, três animais ainda permaneceram com a forma difásica $(-/+)$ e os demais retornaram ao normal. Já na prova $\mathrm{P} 2$, todos os equinos apresentaram a forma bífida $(+/+)$ no repouso, e seis, a forma difásica $(-/+)$ logo após o exercício, sendo que dois ainda permaneceram com esta $30 \mathrm{~min}$ após. A onda $T$ foi a forma mais variável. Houve uma tendência de inversão da polaridade da onda $T$ na maioria dos animais, tornando-se negativa após 0 exercício.

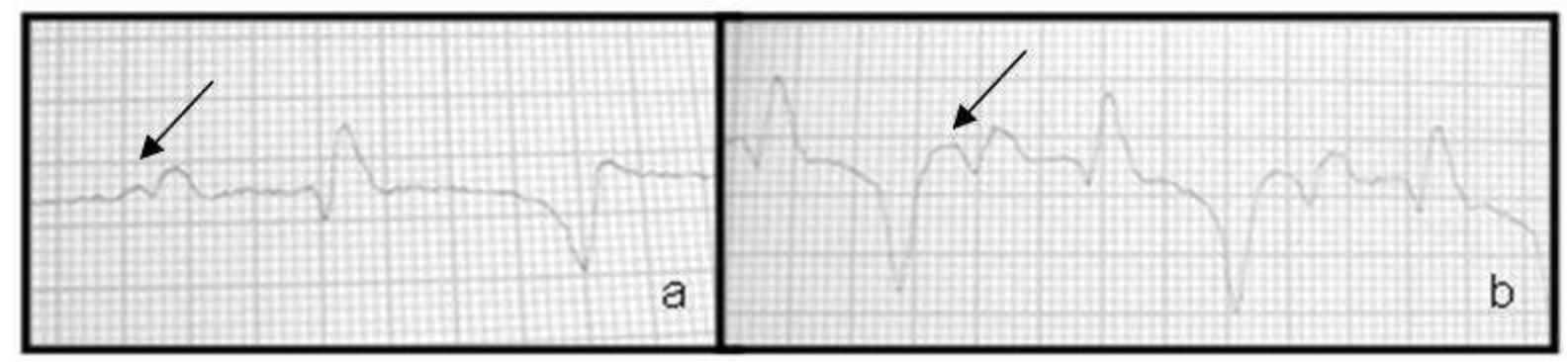

Figura 1 - Morfologia da onda $\mathrm{P}$ (seta): bífida antes do exercício (a) e difásica imediatamente após o exercício. Derivação DII, velocidade $50 \mathrm{~mm} / \mathrm{s}$, sensibilidade N. 
Tabela 1 - Médias ( \pm desvios-padrão) da frequência cardíaca, duração de $P$, amplitude de PII, intervalo PR, duração de QRS, amplitude de R, intervalo QT e duração da onda $T$, e medianas da amplitude de $\mathrm{PI}$ e de $\mathrm{Q}$, em oito equinos da raça Puro Sangue Árabe, nas provas P1 (antes do treinamento) e P2 (após o treinamento) de exercício progressivo em esteira, no repouso (M0), imediatamente após (PE) e 30 min após o exercício

\begin{tabular}{|c|c|c|c|c|c|}
\hline \multirow{2}{*}{$\begin{array}{c}\text { Parâmetros } \\
\text { eletrocardiográficos }\end{array}$} & \multirow{2}{*}{ Prova } & \multicolumn{3}{|c|}{ Momentos } & \multirow[b]{2}{*}{$P$} \\
\hline & & M0 & PE & $30 \mathrm{~min}$ & \\
\hline \multirow{2}{*}{ Frequência cardíaca (bpm) } & P1 & $43,1 \pm 5,9^{A a}$ & $88,1 \pm 9,9^{b}$ & $62,5 \pm 16,7^{c}$ & $<0,0001$ \\
\hline & P2 & $35,6 \pm 4,2^{\mathrm{Ba}}$ & $91,3 \pm 11,3^{b}$ & $62,5 \pm 15,7^{\mathrm{C}}$ & $<0,0001$ \\
\hline$P$ & & $<0,01$ & $>0,5$ & $>1,0$ & \\
\hline \multirow{2}{*}{ Duração de P (s) } & P1 & $0,097 \pm 0,022$ & $0,092 \pm 0,016$ & $0,099 \pm 0,018$ & $>0,7$ \\
\hline & P2 & $0,114 \pm 0,018$ & $0,097 \pm 0,020$ & $0,095 \pm 0,019$ & $>0,1$ \\
\hline$P$ & & $>0,1$ & $>0,5$ & $>0,6$ & \\
\hline \multirow{2}{*}{ Amplitude de PI (mV) } & P1 & 0,1232 & $-0,0941$ & 0,1149 & $>0,16$ \\
\hline & P2 & 0,1295 & $-0,1086$ & 0,1087 & $>0,06$ \\
\hline$P$ & & $>0,9$ & $>0,5$ & $>0,8$ & \\
\hline \multirow{2}{*}{ Amplitude de PII (mV) } & P1 & $0,237 \pm 0,035$ & $0,304 \pm 0,092$ & $0,202 \pm 0,077$ & $>0,06$ \\
\hline & P2 & $0,258 \pm 0,060$ & $0,310 \pm 0,082$ & $0,264 \pm 0,100$ & $>0,4$ \\
\hline$P$ & & $>0,4$ & $>0,9$ & $>0,2$ & \\
\hline \multirow{2}{*}{ Intervalo PR (s) } & P1 & $0,239 \pm 0,031^{a}$ & $0,205 \pm 0,028^{b}$ & $0,214 \pm 0,029^{a b}$ & $<0,05$ \\
\hline & P2 & $0,270 \pm 0,035^{\mathrm{a}}$ & $0,207 \pm 0,027^{\mathrm{b}}$ & $0,229 \pm 0,032^{b}$ & $<0,003$ \\
\hline$P$ & & $>0,09$ & $>0,8$ & $>0,3$ & \\
\hline \multirow{2}{*}{ Duração de QRS (s) } & $\mathrm{P} 1$ & $0,107 \pm 0,030$ & $0,107 \pm 0,028$ & $0,111 \pm 0,031$ & $>0,9$ \\
\hline & P2 & $0,110 \pm 0,029$ & $0,103 \pm 0,028$ & $0,114 \pm 0,034$ & $>0,8$ \\
\hline$P$ & & $>0,7$ & $>0,8$ & $>0,9$ & \\
\hline \multirow{2}{*}{ Amplitude de Q $(\mathrm{mV})$} & P1 & 0,2668 & 0,3793 & 0,3129 & $>0,7$ \\
\hline & P2 & 0,2690 & 0,2649 & 0,3105 & $>0,7$ \\
\hline$P$ & & $>1,0$ & $>0,6$ & $>0,9$ & \\
\hline \multirow{2}{*}{ Amplitude de R (mV) } & P1 & $0,553 \pm 0,246$ & $0,600 \pm 0,300$ & $0,502 \pm 0,198$ & $>0,8$ \\
\hline & P2 & $0,623 \pm 0,220$ & $0,522 \pm 0,215$ & $0,434 \pm 0,283$ & $>0,3$ \\
\hline$P$ & & $>0,6$ & $>0,6$ & $>0,6$ & \\
\hline \multirow{2}{*}{ Intervalo QT (s) } & $\mathrm{P} 1$ & $0,363 \pm 0,057^{a}$ & $0,291 \pm 0,043^{b}$ & $0,320 \pm 0,046^{a b}$ & $<0,02$ \\
\hline & P2 & $0,379 \pm 0,043^{a}$ & $0,262 \pm 0,054^{b}$ & $0,332 \pm 0,043^{b}$ & $<0,0001$ \\
\hline$P$ & & $>0,5$ & $>0,3$ & $>1,0$ & \\
\hline \multirow{2}{*}{ Duração de T (s) } & $\mathrm{P} 1$ & $0,096 \pm 0,044$ & $0,116 \pm 0,059$ & $0,121 \pm 0,036$ & $>0,5$ \\
\hline & P2 & $0,111 \pm 0,034$ & $0,125 \pm 0,080$ & $0,118 \pm 0,025$ & $>0,8$ \\
\hline$P$ & & $>0,4$ & $>0,8$ & $>0,8$ & \\
\hline
\end{tabular}

Letras minúsculas distintas na mesma linha e letras maiúsculas distintas na mesma coluna indicam diferença estatística entre si $(P<0,05)$.

Somente três equinos (animais 8, 15 e 16) apresentaram contrações ventriculares prematuras (VPC) monomórficas e isoladas imediatamente após o exercício. $\mathrm{O}$ animal 8 exibiu dois VPC imediatamente após o exercício somente na prova P1. O equino 15 apresentou três VPC logo após e um em 30 min após o exercício da prova P2. O equino 16 apresentou um VPC em $\mathrm{P} 1$, e quatro em P2, logo após o exercício em ambas as provas.

\section{DISCUSSÃO}

Os valores dos parâmetros observados antes do período de treinamento (P1), no repouso (M0), são similares aos da literatura referentes à raça Puro Sangue Árabe citados por Yonezawa et al. (2007) em 20 equinos sem treinamento, embora 0 intervalo QT seja maior $(0,3534 \pm 0,0497$ s). Os valores obtidos após o treinamento são semelhantes aos encontrados por 


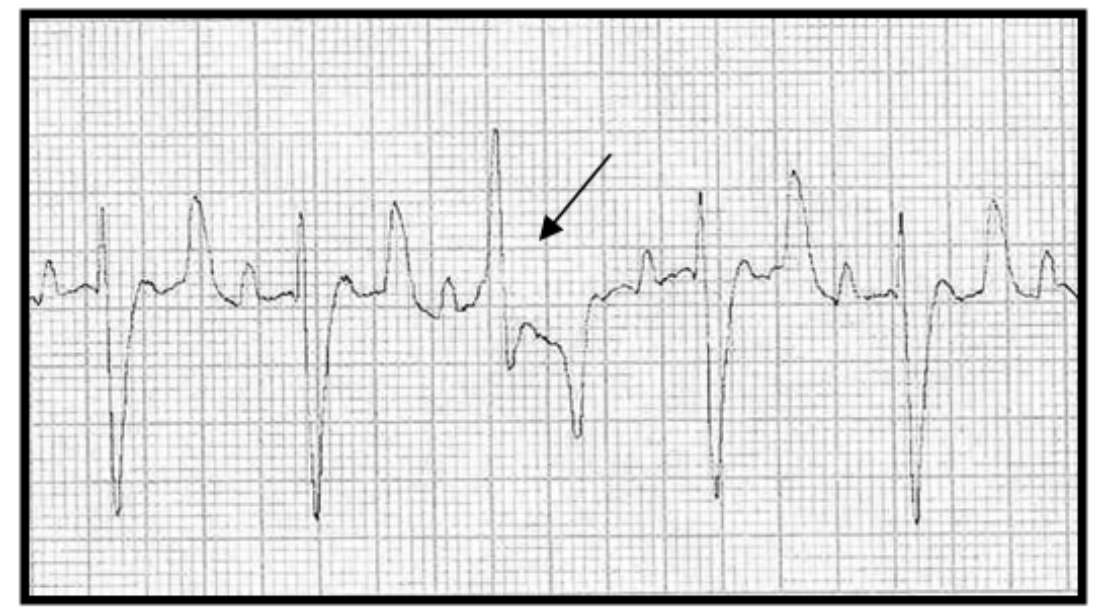

Figura 2 - Contração ventricular prematura (seta) observada no animal 16 imediatamente após o exercício. Derivação DII, velocidade $25 \mathrm{~mm} / \mathrm{s}$, sensibilidade $\mathrm{N}$.

Fazio et al. (2003) em equinos de trote submetidos à corrida de $2.000 \mathrm{~m}$, apesar da duração de QRS $(0,070 \pm 0,0222 \mathrm{~s})$ seja visivelmente maior.

Os resultados indicam que os parâmetros eletrocardiográficos não retornaram aos valores normais de repouso $30 \mathrm{~min}$ após o término do exercício nas duas provas, o mesmo foi observado por Chabchoub et al. (1999) em equinos da raça Puro Sangue Árabe de corrida e por Piccione et al. (2003) em equinos de salto.

A frequência cardíaca é determinada pelo equilíbrio entre as atividades simpática e parassimpática, por catecolaminas e temperatura corpórea (Hamlin et al., 1972). Em P1 e P2, aumentou com o exercício, como observado por Piccione et al. (2003), em equinos de salto. Estes autores atribuíram o estímulo do tônus simpático relacionado à maior frequência cardíaca necessária para acrescer o débito cardíaco resultante do esforço físico.

Muñoz et al. (1995) verificaram a redução da frequência cardíaca de repouso após o treinamento, em equinos da raça Andaluz, assegurando que esta diminuição seja devido ao treinamento e ao menor grau de excitação dos animais quando entram em contato com as pessoas. Neste estudo, observou-se uma menor frequência cardíaca em P2, mas sugere-se que seja devido ao manejo dos animais, uma vez que não houve diminuição durante a fase de recuperação após o exercício.

Em relação ao intervalo $P R$, houve diminuição significativa após o exercício, como reportado por alguns autores (Senta et al., 1970; Fazio et al., 2003; Piccione et al., 2003), que pode ser explicado pelo aumento da frequência cardíaca. $O$ intervalo QT diminuiu significativamente com o efeito do exercício físico. Além disso, apresentou-se menor que o valor de repouso durante o período de recuperação (30 min). Senta et al. (1970) observaram o mesmo, justificando que provavelmente houve influência dos nervos autonômicos na permeabilidade da membrana celular, alterações eletrolíticas, temperatura e hipóxia da musculatura ventricular, mas não identificou os fatores primariamente responsáveis pelo intervalo QT mais curto na recuperação.

A morfologia da onda $\mathrm{P}$ bífida $(+/+)$ observada na maioria dos animais em repouso é um achado normal no eletrocardiograma do Puro Sangue Inglês, do American Trotter (Fregin, 1982), do Puro Sangue Árabe (Yonezawa et al., 2007), do Mangalarga (Vicenzi et al., 2000) e de equinos da raça Espanhola (Ayala et al., 2000), podendo ser dividida em componentes I (PI) e II (PII). Esse fenômeno pode ser atribuído aos diferentes pontos de ativação do átrio, 
confirmados por meio da vetocardiografia (Hamlin et al., 1970). Senta et al. (1970), em equinos de trote, e Ayala et al. (2000), em equinos da raça Espanhola, descreveram que o aumento da frequência cardíaca durante o exercício fez com que houvesse a fusão dos dois componentes e, assim, a onda $\mathrm{P}$ assumiu um contorno monofásico positivo. Em contraste, neste estudo observou-se a inversão da polaridade, sendo que a onda $P$ apresentou-se difásica (-/+). Esta alteração pode ser explicada pelo estímulo do átrio direito posterior perto do seio coronário (Hamlin et al., 1970).

A onda $\mathrm{T}$ compreende o parâmetro mais variável do eletrocardiograma (Holmes e Rezakhani, 1975). Sua interpretação é muito difícil por variar de forma e amplitude (Sevestre, 1982). Ayala et al. (2000) também observaram a inversão da polaridade da onda $\mathrm{T}$ em equinos da raça Espanhola, submetidos a exercício extenuante. A taquicardia transitória parece afetar a direção desta onda, sugerindo que o processo de repolarização no miocárdio ventricular deva ser afetado pela duração da diástole e fatores que afetam essa duração, incluindo efeitos autonômicos e possívelmente os metabólicos nas fibras miocárdicas influenciadas pela circulação coronariana (Holmes e Rezakhani, 1975).

Os animais utilizados eram clinicamente sadios e não apresentavam alterações eletrocardiográficas de repouso que pudessem interferir no desempenho. Mesmo assim, pôde-se constatar a ocorrência de VPC isolados em três animais após o exercício, ocorrendo somente durante a recuperação do exercício, entre logo após e 30 min após o exercício. Senta et al. (1970) acreditam que os VPC desse tipo, após atividade física, são funcionais e induzidos pelo reajuste do tônus nervoso autonômico e, portanto, benignos.

\section{CONCLUSÃO}

O eletrocardiograma mostrou-se eficiente na avaliação das alterações cardíacas promovidas pelo exercício físico. Entretanto, são necessários mais estudos para elucidar o significado clínico dessas alterações observadas. A suplementação de vitamina $E$ nestas condições não influenciou os parâmetros eletrocardiográficos. A redução da frequência cardíaca após o treinamento foi atribuída ao manejo dos animais e, sendo assim, não houve efeito considerável do treinamento sobre o eletrocardiograma.

\section{AGRADECIMENTOS}

Os autores agradecem à Fundação de Amparo à Pesquisa do Estado de São Paulo (FAPESP) pelo apoio financeiro concedido.

Projeto aprovado pela Câmara de Ética em Experimentação Animal (protocolo no 116/2005-CEEA).

\section{REFERÊNCIAS}

ASCENSÃO, A.; MAGALHÃES, J.; SOARES, J. et al. Exercício e stress oxidativo cardíaco. Revista Portuguesa de Cardiologia, v.22, n.5, p.651-678, 2003.

AYALA, I.; GUTIERREZ-PANIZO, C.; BENEDITO, J.L. et al. Morphology and amplitude values of the electrocardiogram of Spanish-bred horses of different ages in the Dubois leads system.

Veterinary Research, v.31, p.347-354, 2000.

CHABCHOUB, A.; LANDOLSI, F.; OUESLATI, S. Comparaison de la fréquence cardiaque et des paramètres hèmatologiques et électrocardiographiques chez le cheval pur-sang arabe performant et non performant em Tunisie. Revue de Médecine Vétérinaire, v.150, p.467-472, 1999.

COSTA, G.; ILLERA, M.; GARCÍA-SACRISTÁN, A. Electrocardiographical values in non-trained horses. Zentralblatt fur Veterinärmedizin Reihe A, v.32, p.196-201, 1985.

DE MOFFARTS, B.; KIRSCHVINK, N.; PINCEMAIL, $\mathrm{J}$. et al. Effect of oral antioxidant supplementation on blood antioxidant status in trained Thoroughbred 
horses. The Veterinary Journal, v.169, p.65-74, 2005.

DOUGLAS, P.; O'TOOLE, M.; DOUGLAS, W. et al. Cardiac fatigue after prolonged exercise.

Circulation, v.76, p.1206-1213, 1987.

DURANDO, M.M.; REEF, V.B.; KLINE, K. et al. Acute effects of short duration maximal exercise on cardiac troponin I in healthy horses. Equine and Comparative Exercise Physiology, v.3, p.217223, 2006.

EVANS, D.L. Cardiac responses to exercise and training. In: MARR, C.M. Cardiology of the horse. Philadelphia : W.B. Saunders, 1999. p.32-46.

FAZIO, F.; FERRANTELLI, V.; PICCIONE, G. et al. Variations in some electrocardiographic parameters in the trotter during racing and training. Veterinary Research Communications, v.27, p.229-232, 2003.

FERNANDES, W.R.; LARSSON, M.H.M.A.; ALVES, A.L.G. et al. Características eletrocardiográficas em equinos clinicamente normais da raça Puro Sangue Inglês. Arquivo Brasileiro de Medicina

Veterinária e Zootecnia, v.56, p.143-149, 2004.

FREGIN, G.F. The equine electrocardiogram with standardized body and limb positions. Cornell Veterinarian, v.72, p.304-324, 1982.

HAMLIN, R.L.; KLEPINGER, W.L.; GILPIN, K.W. et al. Autonomic control of heart rate in the horse.

American Journal of Physiology, v.222, p.976978, 1972.

HAMLIN, R.L.; SMETZER, D.L.; SENTA, T. Atrial activation paths and $P$ waves in horses. American Journal of Physiology, v.219, p.306-313, 1970.

HOLMES, J.R.; REZAKHANI, A. Observations on the T wave of the equine electrocardiogram. Equine Veterinary Journal, v.7, p.55-62, 1975.

JESTY, A.S.; REEF, V.B. Evaluation of the horse with acute cardiac crisis. Clinical Techniques in Equine Practice, v.5, p.93-103, 2006.

$\mathrm{KLEI}, \mathrm{T}$.R. Parasite controls programs. In: ROBINSON, N.E. Current therapy in equine medicine, 4 ed. Philadelphia : W.B. Saunders, 1997. p.709-713.

KIRYU, K.; MACHIDA, N.; KASHIDA, Y. et al. Pathologic and electrocardiographic findings in sudden cardiac death in racehorses. Journal of Veterinary Medicine Science, v.61, p.921-928, 1999.

LEROUX, A.J.; SCHOTT, H.C.; HINES, M.T. Ventricular tachycardia associated with exhaustive exercise in a horse. Journal of American

Veterinary Medical Association, v.207, p.335-337, 1995.
LEWIS, L.D. Nutrição clínica equina: alimentação e cuidados. São Paulo : Roca, 2000. 710p.

MACHADO, L.P.; SAITO, M.E.; SILVEIRA, V.F. et al. Susceptibilidade eritrocitária ao estresse osmótico em equinos da raça Árabe: efeito do exercício, treinamento e suplementação com vitamina E. Revista Universidade Rural, Série Ciências da Vida, Suplemento, v.27, p.134-136, 2007.

MARTIN, B.B.; REEF, V.B.; PARENTE, E.J. et al. Causes of poor performance of horses during training, racing, or showing: 348 cases (1992-1996). Journal of the American Veterinary Medical Association, v.216, p.554-558, 2000.

MUÑOZ, A.; CASTEJÓN, F.; RUBIO, M.D. et al. Electrocardiographic alterations in Andalusian horses associated with training. Journal of Equine Veterinary Science, v.15, p.72-79, 1995.

NEUMAYR, G.; PFISTER, R.; MITTERBAUER, G. et al. Effect of the "Race across the Alps" in elite cyclists on plasma cardiac troponins I and T.

American Journal of Cardiology, v.89, p.484-486, 2002.

PICCIONE, G.; ASSENZA, A.; FAZIO, F. et al. Electrocardiographic changes induced by physical exercise in the jumper horse. Arquivo Brasileiro de Medicina Veterinária e Zootecnia, v.55, p.397404, 2003.

REEF, V.B. Ambulatory and exercise electrocardiography and post-exercise echocardiography. In: MARR, C.M. Cardiology of the horse. Philadelphia : W.B. Saunders, 1999. p.150-160.

\section{SAMPAIO, I.B.M. Estatística aplicada à} experimentação animal. 1ed. Belo Horizonte : UFMG, 1998. 221p.

SENTA, T.; SMETZER, D.L.; SMITH, C.R. Effects of exercise on certain eletrocardiographic parameters and cardiac arrhythmias in the horse. A radiotelemetric study. Cornell Veterinarian, v.60, p.552-569, 1970.

SEVESTRE, J. A eletrocardiografia no cavalo. A Hora Veterinária, v.2, p.28-36, 1982.

SIEGEL, S. Estatística não-paramétrica. São Paulo : McGraw-Hill, 1975. 350 p.

SKARDA, R.T.; MUIR, W.W.; MILNE, D.W. et al. Effects of training on resting and postexercise ECG in Standardbred horses, using a standardized exercise test. American Journal of Veterinary Research, v.37, p.1485-1488, 1976.

VENDITTI, P.; MASULLO, P.; DI MEO, S. Et al. Protection against ischemia-reperfusion induced oxidative stress by vitamin $E$ treatment. Archives of 
Physiology and Biochemistry, v.107, p.27-34, 1999.

VICENZI, R.C.; LARSSON, M.H.M.A.;

FERNANDES, W.R. Parâmetros

eletrocardiográficos em equinos clinicamente

normais da raça Mangalarga. Parte III: amplitude e duração dos complexos e intervalos. Revista

Brasileira de Medicina Veterinária, v.22, p.194198, 2000.

WHITE, N.A.; RHODE, E.A. Correlation of electrocardiographic findings to clinical disease in the horse. Journal of American Veterinary Medical Association, v.164, p.46-56, 1974.
WHYTE, G.P.; GEORGE, K.; SHARMA, S. et al. Cardiac fatigue following prolonged endurance exercise of differing distances. Medicine and Science in Sports and Exercise, v.32, p.10671072, 2000.

YONEZAWA, L.A.; SILVEIRA, V.F.; MACHADO, L.P. et al. Alguns parâmetros eletrocardiográficos em equinos da raça Árabe. Revista Universidade Rural, Série Ciências da Vida, Suplemento, v.27, p.78-79, 2007. 